Professor of Pathology, Harvard Medical School, Bader 124.1, 300 Longwood Ave., Boston, Massachusetts 02115, USA. Phone: 617.919.2664; Fax: 617.730.0168; E-mail: mark.fleming@childrens.harvard.edu.

1. Knutson M, Wessling-Resnick M. Iron metabolism in the reticuloendothelial system. Crit Rev Biochem Mol Biol. 2003;38(1):61-88.

2. Severance S, Hamza I. Trafficking of heme and porphyrins in metazoa. Chem Rev. 2009; 109(10):4596-4616.

3. Chiabrando D, et al. The mitochondrial heme exporter FLVCR1b mediates erythroid differentiation. J Clin Invest. 2012;122(12):4569-4579.

4. Uzel C, Conrad ME. Absorption of heme iron. Semin Hematol. 1998;35(1):27-34.

5. Yanatori I, Tabuchi M, Kawai Y, Yasui Y, Akagi R, Kishi F. Heme and non-heme iron transporters in non-polarized and polarized cells. BMC Cell Biol. 2010;11:39.

6. Donovan A, et al. Positional cloning of zebrafish ferroportin 1 identifies a conserved vertebrate iron exporter. Nature. 2000;403(6771):776-781.

7. Nemeth E, et al. Hepcidin regulates cellular iron efflux by binding to ferroportin and inducing its internalization. Science. 2004;306(5704):2090-2093.

8. Qiu A, et al. Identification of an intestinal folate transporter and the molecular basis for hereditary folate malabsorption. Cell. 2006;127(5):917-928.

9. Salojin KV, et al. A mouse model of hereditary folate malabsorption: deletion of the PCFT gene leads to systemic folate deficiency. Blood. 2011; 117(18):4895-4904

10. Krishnamurthy $P$, et al. The stem cell marker Bcrp/ABCG2 enhances hypoxic cell survival through interactions with heme. J Biol Chem. 2004; 279(23):24218-24225.

11. Robey RW, Ierano C, Zhan Z, Bates SE. The challenge of exploiting ABCG2 in the clinic. Curr Pharm Biotechnol. 2011;12(4):595-608.

12. Saison C, et al. Null alleles of ABCG2 encoding the breast cancer resistance protein define the new blood group system Junior. Nat Genet. 2012; 44(2):174-177.

13. Rajagopal A, et al. Haem homeostasis is regulated by the conserved and concerted functions of HRG-1 proteins. Nature. 2008;453(7198):1127-1131.

14. Yuan X, Protchenko O, Philpott CC, Hamza I. Topologically conserved residues direct heme transport in HRG-1-related proteins. J Biol Chem.
2012;287(7):4914-4924.

15. Delaby C, et al. Subcellular localization of iron and heme metabolism related proteins at early stages of erythrophagocytosis. PloS One. 2012;7(7):e42199.

16. Quigley JG, et al. Cloning of the cellular receptor for feline leukemia virus subgroup C (FeLV-C), a retrovirus that induces red cell aplasia. Blood. 2000;95(3):1093-1099.

17. Khan AA, Quigley JG. Control of intracellular heme levels: heme transporters and heme oxygenases. Biochim Biophys Acta. 2011;1813(5):668-682.

18. Keel SB, et al. A heme export protein is required for red blood cell differentiation and iron homeostasis. Science. 2008;319(5864):825-828.

19. Quigley JG, Gazda H, Yang Z, Ball S, Sieff CA, Abkowitz JL. Investigation of a putative role for FLVCR, a cytoplasmic heme exporter, in Diamond-Blackfan anemia. Blood Cells Mol Dis. 2005; 35(2):189-192.

20. Ishiura $\mathrm{H}$, et al. Posterior column ataxia with retinitis pigmentosa in a Japanese family with a novel mutation in FLVCR1. Neurogenetics. 2011; 12(2):117-121.

21. Rajadhyaksha AM, et al. Mutations in FLVCR1 cause posterior column ataxia and retinitis pigmentosa. Am J Hum Genet. 2010;87(5):643-654.

\title{
Taking a bite: endocytosis in the maintenance of the slit diaphragm
}

\author{
Rosemary V. Sampogna and Qais Al-Awqati
}

Department of Medicine, College of Physicians and Surgeons of Columbia University, New York, New York, USA.

\begin{abstract}
In the kidney, the slit diaphragm joins adjacent podocytes, forming an epithelial barrier that filters plasma into the urinary space, yet retains blood cells and proteins within the circulation. In this issue of the JCI, Soda et al. have identified clathrin-mediated endocytosis as a central mechanism by which the function and structural integrity of the slit diaphragm are maintained.
\end{abstract}

The glomeruli of the human kidney filter almost 200 liters of fluid every day, but must retain the albumin and other large proteins within the circulation. The fluid has to cross three layers: the endothelium, the glomerular basement membrane (GBM), and the epithelial cells or podocytes. The GBM (1) and the slit diaphragm (2), a structure that joins adjacent podocytes, are two barriers to protein filtration, but the endothelial cell also likely plays a role. Disruption of these barriers results in proteinuria, a consequence of many kidney diseases.

Podocytes begin life as tall epithelial cells that have typical tight junctions near the apex separating the apical surface from the lateral cell membranes. As development

Conflict of interest: The authors have declared that no conflict of interest exists.

Citation for this article: J Clin Invest. 2012; 122(12):4330-4333. doi:10.1172/JCI65785. progresses, these junctions migrate downward toward the basal surface of the mature podocytes, becoming the slit diaphragm (3). Unlike other polarized epithelia, the lateral surface is above these specialized tight junctions and is continuous with and identical to the apical surface. The basal surface differentiates into thin interdigitating structures called foot processes that are joined together by the cell-cell junctions of the slit diaphragm. During proteinuric disease, the foot processes lose their fine structure and collapse into a mass of cytoplasm on top of the basement membrane (4). The observation that a great abundance of actin and actin-binding proteins accumulates in these diseased foot processes suggested that actin dysregulation might play a key role in the pathogenic progression (5). The discovery that $\alpha$-actinin 4 mutations were also associated with heavy proteinuria also helped bring attention to the role of actin (6).
Since then, mutations in humans or animals in other actin-binding proteins including Nck, CD2AP, and dynamin were also found to mediate proteinuria (7). Further, the cytoplasmic domains of many slit diaphragm components can bind actin or signal to actin, further suggesting a key role for this cytoskeletal protein (8). But what was the actual cell biological process in which actin was so central? Was the primary function of actin to maintain the slit diaphragm, or was the actin rearrangement merely a consequence of the permeability change? Here, one might turn to basic cell biology and physiology to find the definitive answers. Unfortunately, the podocyte, when grown in culture, loses its complex shape; it has neither foot processes nor a real slit diaphragm. Given that actin is directly involved in cell shape determination and maintenance, this has placed the field in a difficult position. However, an article in this issue by Soda et al. has provided the first insight into the process of maintenance of the slit diaphragm (9). The authors show that clathrinmediated endocytosis is required for podocyte function and is the likely effector of the changes in actin that are so often observed. 


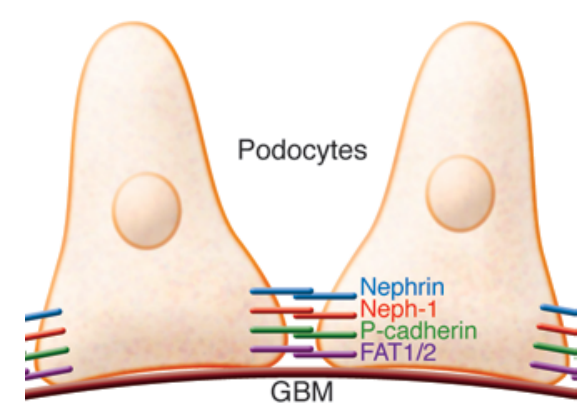

Figure 1

Podocytes and the slit diaphragm. Schematic of neighboring podocytes attached to the GBM, with key cell-cell adhesion proteins, including nephrin, Neph-1, P-cadherin, and the protocadherins FAT1 and FAT2.

\section{The slit diaphragm is a specialized epithelial junction}

Our understanding of the molecular components of the slit diaphragm benefited from advances in positional cloning, which identified nephrin as the founding member of this protein complex. Other proteins were then rapidly discovered, including Neph-1 (a homologous protein to nephrin), podocin, P-cadherin, and the protocadherins FAT1 and FAT2 (ref. 2 and Figure 1). The cadherins bind, on their cytoplasmic end, to $\alpha, \beta$, and $\gamma$ catenins. These studies led to the suggestion that the slit diaphragm is a unique adherens junction, rather than a tight junction (10). The finding that $\mathrm{ZO}-1$, the classic tight junction scaffolding protein, was present in the slit diaphragm suggested that the structure is a modified tight junction. More recent studies showed that typical tight junction proteins, such as occludins, JAM-A, and cingulin, also exist in the slit diaphragm (11). Remarkably, in experimental nephrosis, more typical tight junctions reappear in the podocytes (12). Thus, the slit diaphragm is clearly a unique cell connector composed of proteins that are typical of two classes of junctions.

\section{Endocytosis regulates slit diaphragm proteins}

Endocytosis using clathrin-coated vesicles occurs in all eukaryotic cells and serves many functions, including recycling of membrane proteins, delivery of material into the cell, regulation of the number of transport proteins, and cytoplasmic signaling (13). It proceeds in five identifiable steps. The first of these is specification of a site where the membrane becomes dimpled, a process that requires the accumulation of patches of phosphatidylinositol- 4,5-bisphosphate. Second, these patches recruit several membrane-bending proteins, and the membrane adaptor protein AP2 binds to a specific "cargo" protein, such as nephrin, in podocytes. Third, the binding of AP2 allows the clathrin coat to be assembled into the typical triskelion cage. Fourth, dynamin, the mechanochemical enzyme powered by GTP hydrolysis, then binds to the "neck" of the vesicle (in association with other proteins, such as endophilins, that have specific dimerization motifs called BAR domains), eventually resulting in scission of the neck. Finally, the neck of the triskelion cage contains a defect that

\section{Figure 2}

allows inositol-5 phosphatases such as synaptojanin to disassemble the clathrin cage.

It was previously known that dynamin degradation can result in proteinuria and foot process effacement, but this was ascribed to the fact that dynamin is also an actin-binding protein (14). Soda et al. now describe the deletion of several proteins critical for clathrin-mediated endocytosis specifically in podocytes (ref. 9 and Figure 2). They found that deletion of synaptojanin, dynamin, or endophilin caused proteinuria and foot process effacement. The limitation to this approach is that any of these proteins might regulate actin directly, and the resultant proteinuria might not be related to an endocytosis defect. However, recent studies have shown that recruitment of dynamin, BAR domain proteins, and endophilin to the site of clathrinmediated endocytosis requires actin, and furthermore that actin and dynamin promote recruitment of one another through a positive feedback system (15). In podocytes in culture, Soda et al. were able to demonstrate that actin accumulates with dynamin in sites of clathrin-mediated endocytosis, but not at sites of actin stress fibers (9). Furthermore, the authors demonstrated that nephrin is endocytosed by clathrincoated vesicles. These studies also clarified the role of other proteins implicated

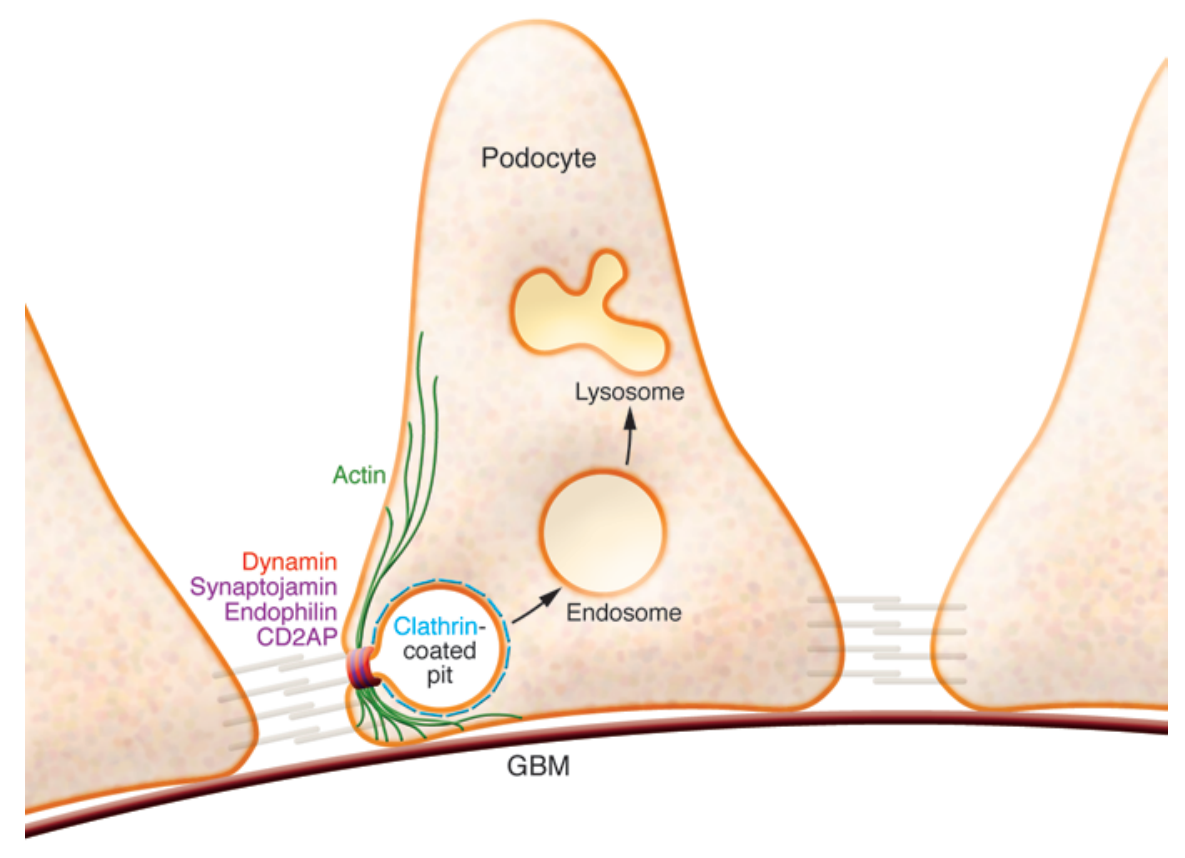

Endocytosis in the podocyte. Assembly of clathrin-coated pits involves recruitment of various membrane-associated proteins, including dynamin and endophilins, which bind at the neck of the forming vesicle; the membrane adaptor protein CD2AP; and the phosphatase synaptojanin. 
in proteinuria and foot process effacement, such as myosin $1 \mathrm{E}$ (which accumulates at sites of endocytosis) and CD2AP (which is a binding partner of endophilin and interacts with dynamin and synaptojanin). Nck is also an adaptor that binds to dynamin and synaptojanin, and PI $3 \mathrm{~K} 2 \alpha$ binds to clathrin and is involved in endocytosis. Although the present studies do not rule out a primary role for actin dynamics in proteinuric disease, they do raise the bar for the kind of evidence that will be needed to document such a mechanism.

\section{Endocytosis in epithelial junctions}

The work of Soda et al. (9) forces us to confront the role of endocytosis in the maintenance and regulation of the slit diaphragm and perhaps all junctional complexes. Significant advances in structural and basic cell biology have led to a clearer understanding of the structure and function of epithelial junctions, but what role could endocytosis be playing? The formation of adherent junction was thought to be made in two steps: delivery of cadherins to the surface resulted in a mobile pool, which then - apparently stabilized by cell-cell cadherin contacts - accumulated first into spots and then into long linear junctions. Advances in video microscopy have demonstrated that tight and adherent junctions are dynamic structures both in vivo and in the established junctions of confluent epithelial cell cultures. de Beco et al. studied "mature" epithelial junctions and found that most E-cadherin molecules did not diffuse in the plane of the membrane, but rather had a rapid turnover that was mediated by endocytosis (16). Blockade of dynamin in this system stopped endocytosis, but did not result in E-cadherin redistribution, which suggests that endocytosis is the major mechanism of stabilization of junction-located cadherin. Endocytosis was also shown to be very active in removal of claudins from tight junctions. In confluent epithelial cultures, Matsuda et al. showed a most startling result, that endocytosis was peculiar (in their words), as the vesicle seemed to "tear" off the claudins (a class of tight junction membrane proteins) of the two apposing cells, resulting in a doublemembrane vesicle that was directed to late endosomes and then presumably lysosomes (17). Further, it was not the whole membrane that was "bitten" off by the endocytic vesicle, but only the patch that harbored claudins, because the doublemembrane vesicles did not contain ZO-1 or
JAM-A. Clearly, the claudins were destined for degradation, because the authors also demonstrated that the E3 ubiquitin ligase LNX1 bound to claudins and caused their removal from the junctional complex (18).

Thus, in all junctions (and we can now include the slit diaphragm in the list), endocytosis is critical for maintenance of permeability and even polarity of the epithelial cell. Endocytosis is very rapid and has been shown to be associated either with recycling of the proteins back to the junction or with processing them for degradation (19). However, many studies were done in cell culture, sometimes following the disruption and reformation of the junction. Other studies of endocytosis in vivo have been usually performed in the context of tissue development (20) or pathological states, such as epithelial-mesenchymal transition and oncogenesis. But what do the present studies tell us about the role of this process in maintenance of mature epithelial structures in vivo? Does vigorous endocytosis imply that junctional proteinprotein contacts become unstable and constantly need renewal? Or, if the purpose of this process is to simply recycle protein components, what might be the advantage of repeatedly making and breaking the junction? It is probable that junctions are always under stress, most likely from the movement of cell membranes against each other. Hence, the strands formed by adhesion proteins might break frequently and require replacement. In the case of the slit diaphragm, the permeability barrier is subjected to the high pulsatile pressure of the glomerular capillary as well as to massive fluid flow through these junctions. These stresses might necessitate continuous replacement of damaged strands of junctional complexes.

The slit diaphragm junction is composed of many adhesion proteins, including P-cadherin, the giant protocadherin FAT, nephrin, Neph-1 and Neph-2, and occludin. While the molecular details remain unclear, there are at present excellent cadherin structural studies (21). These reveal that swapping of $\beta$-strand domains located on cadherins belonging to two neighboring epithelial cells forms extremely tight adhesive cell-cell interactions. These might provide key stabilizing interactions that maintain the structural integrity of the slit diaphragm. This is particularly notable in the kidney, given that all components of the glomerular permeability barrier are continually exposed to significant mechanical and shear stress as filtrate is forced into the urinary space in a pulsatile manner. There is also good evidence that classical cadherins act as mechanosensors to sense and transmit these forces. For instance, endothelial cells were shown to adapt to shear forces exerted by blood flow via a network that included VE-cadherin, PECAM, and integrins. Activation of VEGFR increased vascular permeability by causing clathrin-mediated endocytosis of VE-cadherin and hence disrupting endothelial cell-cell junctions (22). Furthermore, application of mechanical force in vitro to E-cadherin elicited proportional changes in junctional adhesion strength (and at the same time required an intact actomyosin skeleton) (23). Finally, it was recently demonstrated that the actomyosin cytoskeleton exerts tension on membranebound E-cadherin that is further increased at cell-cell junctions when adhering cells are stretched (24).

\section{Conclusions}

The present findings of Soda et al. (9) contribute to accumulating evidence that podocyte cell-cell junctions are not static structures in a stable environment. Rather, constant fluctuation in systemic blood pressure, salt intake, and intravascular volume likely require continual remodeling, fine tuning, and repair of slit diaphragm substructures. Clathrin-mediated endocytosis would provide a rapid and efficient mechanism to degrade, recycle, and/or regulate the number of podocyte adherens junctions in response to physiologic conditions. Moreover, cadherin-based sensing of dynamic forces in the glomerular environment and regulation of intracellular signaling poses an intriguing mode of mechanotransduction. It is perhaps fortunate that the human P-cadherin situated in the slit diaphragm has an unusually tight binding affinity relative to most other vertebrate P-cadherins (B. Honig, unpublished observations), given our own dietary affinity for salt.

\section{Acknowledgments}

R.V. Sampogna is the recipient of a Carl W. Gottschalk Research Scholar Grant from the American Society of Nephrology.

Address correspondence to: Qais Al-Awqati, Department of Medicine, College of Physicians and Surgeons of Columbia University, 630 W. 168th St., New York, New York 10032, USA. Phone: 212.305.3512; Fax: 212.305.3475; E-mail: qa1@columbia.edu. 
1. Farquhar MG. The glomerular basement membrane: not gone, just forgotten. J Clin Invest. 2006; 116(8):2090-2093.

2. Garg P, Holzman LB. Podocytes: gaining a foothold. Exp Cell Res. 2012;318(9):955-963.

3. Reeves W, Caulfield JP, Farquhar MG. Differentiation of epithelial foot processes and filtration slits: sequential appearance of occluding junctions, epithelial polyanion, and slit membranes in developing glomeruli. Lab Invest. 1978;39(2):90-100.

4. Farquhar MG, Vernier RL, Good RA. An electron microscope study of the glomerulus in nephrosis, glomerulonephritis, and lupus erythematosus. J Exp Med. 1957;106(5):649-660.

5. Shirato I, Sakai T, Kimura K, Tomino Y, Kriz W. Cytoskeletal changes in podocytes associated with foot process effacement in Masugi nephritis. AmJ Pathol. 1996;148(4):1283-1296.

6. Kaplan JM, et al. Mutations in ACTN4, encoding alpha-actinin-4, cause familial focal segmental glomerulosclerosis. Nat Genet. 2000;24(3):251-256.

7. Faul C, Asanuma K, Yanagida-Asanuma E, Kim K, Mundel P. Actin up: regulation of podocyte structure and function by components of the actin cytoskeleton. Trends Cell Biol. 2007;17(9):428-437.

8. Verma R, Kovari I, Soofi A, Nihalani D, Patrie K, Holzman LB. Nephrin ectodomain engagement results in Src kinase activation, nephrin phosphorylation, Nck recruitment, and actin polymerization. J Clin Invest. 2006;116(5):1346-1359.
9. Soda $\mathrm{K}$, et al. Role of dynamin, synaptojanin, and endophilin in podocyte foot processes. J Clin Invest. 2012;122(12):4401-4411.

10. Reiser J, Kriz W, Kretzler M, Mundel P. The glomerular slit diaphragm is a modified adherens junction. J Am Soc Nephrol. 2000;11(1):1-8.

11. Fukasawa H, Bornheimer S, Kudlicka K, Farquhar MG. Slit diaphragms contain tight junction proteins. J Am Soc Nephrol. 2009;20(7):1491-1503.

12. Caulfield JP, Reid JJ, Farquhar MG. Alterations of the glomerular epithelium in acute aminonucleoside nephrosis. Evidence for formation of occluding junctions and epithelial cell detachment. $L a b$ Invest. 1976;34(1):43-59.

13. McMahon HT, Boucrot E. Molecular mechanism and physiological functions of clathrin-mediated endocytosis. Nat Rev Mol Cell Biol. 2011;12(8):517-533.

14 . Sever $\mathrm{S}$, et al. Proteolytic processing of dynamin by cytoplasmic cathepsin $\mathrm{L}$ is a mechanism for proteinuric kidney disease. J Clin Invest. 2007; 117(8):2095-2104.

15. Taylor MJ, Lampe M, Merrifield CJ. A feedback loop between dynamin and actin recruitment during clathrin-mediated endocytosis. PLoS Biol. 2012;10(4):e1001302.

16. de Beco S, Gueudry C, Amblard F, Coscoy S. Endocytosis is required for $\mathrm{E}$-cadherin redistribution at mature adherens junctions. Proc Natl Acad Sci US A. 2009;106(17):7010-7015.

17. Matsuda M, Kubo A, Furuse M, Tsukita S. A pecu- liar internalization of claudins, tight junctionspecific adhesion molecules, during the intercellular movement of epithelial cells. J Cell Sci. 2004; 117(pt 7):1247-1257.

18. Takahashi S, et al. The E3 ubiquitin ligase LNX1p 80 promotes the removal of claudins from tight junctions in MDCK cells. J Cell Sci. 2009;122(pt 7):985-994.

19. Ivanov AI, Nusrat A, Parkos CA. Endocytosis of the apical junctional complex: mechanisms and possible roles in regulation of epithelial barriers. Bioessays. $2005 ; 27(4): 356-365$.

20. Levayer R, Pelissier-Monier A, Lecuit T. Spatial regulation of Dia and Myosin-II by RhoGEF2 controls initiation of E-cadherin endocytosis during epithelial morphogenesis. Nat Cell Biol. 2011;13(5):529-540.

21. Brasch J, Harrison OJ, Honig B, Shapiro L. Thinking outside the cell: how cadherins drive adhesion. Trends Cell Biol. 2012;22(6):299-310.

22. Gavard J, Gutkind JS. VEGF controls endothelialcell permeability by promoting the beta-arrestindependent endocytosis of VE-cadherin. Nat Cell Biol. 2006;8(11):1223-1234.

23. de Beco S, Gueudry C, Amblard F, Coscoy S. Endocytosis is required for E-cadherin redistribution at mature adherens junctions. Proc Natl Acad Sci US A. 2009;106(17):7010-7015.

24. Klink TA, et al. Development and validation of a genericfluorescent methyltransferase activity assay based on the transcreener AMP/GMP assay. J Biomol Screen. 2012;17(1):59-70.

\section{Leaky channels make weak muscles}

\section{Alfred L. George Jr.}

Departments of Medicine and Pharmacology, Vanderbilt University, Nashville, Tennessee, USA.

\begin{abstract}
Mutations in the skeletal muscle voltage-gated calcium channel (Cav1.1) have been associated with hypokalemic periodic paralysis, but how the pathogenesis of this disorder relates to the functional consequences of mutations was unclear. In this issue of the JCI, Wu and colleagues recapitulate the disease by generating a novel knock-in Cav1.1 mutant mouse and use this model to investigate the cellular and molecular features of pathogenesis. They demonstrated an aberrant muscle cell current conducted through the $\mathrm{Ca}_{v} 1.1$ voltage-sensor domain (gating pore current) that explains an abnormally depolarized muscle membrane and the failure of muscle action potential firing during challenge with agents known to provoke periodic paralysis. Their work advances understanding of molecular and cellular mechanisms underlying an inherited channelopathy.
\end{abstract}

Ion channels are ubiquitous membrane proteins that confer selective ionic permeability to the plasmalemma or intracellular membranes and enable a wide variety of important physiological processes, including membrane excitability, synaptic transmission, signal transduction, cell volume regulation, and transcellular ion transport. The vital nature of ion channels is reflected by the existence of inherited disorders

Conflict of interest: The author has declared that no conflict of interest exists.

Citation for this article: J Clin Invest. 2012; 122(12):4333-4336. doi:10.1172/JCI66535. caused by mutations in genes that encode these proteins (1-5). These "channelopathies" represent more than 50 human genetic diseases, including several affecting skeletal muscle contraction, such as the periodic paralyses and nondystrophic myotonias (see "Muscle channelopatbies").

\section{Hypokalemic periodic paralysis}

Plasma membrane channels in skeletal muscle are essential for the generation and propagation of action potentials, leading to release of intracellular calcium through the process of excitation-contraction coupling. Ion channel dysfunction can hinder contraction by impairing action potential firing along the membrane. A characteristic symptom of this phenomenon is known as "periodic paralysis," a form of paroxysmal weakness that occurs in the absence of neuromuscular junction or motor neuron disease. Periodic paralysis is most often thought of as an inherited disease, but certain acquired conditions can produce a similar phenotype. Disturbances in plasma potassium ion concentration often accompany bouts of weakness, and the direction of change has been used to classify the condition as hypokalemic, hyperkalemic, or normokalemic periodic paralysis (6). These conditions are not lethal, because respiratory muscles are spared.

Although the clinical features and inheritance pattern have been known since the first half of the twentieth century, the pathophysiology of periodic paralysis remained mysterious until approximately 30 years ago, when investigators in Germany published their electrophysiological observations on explanted intercostal muscle fibers from subjects with periodic paralysis (7). These studies revealed that resting membrane potential was less nega- 